\title{
Internalization of the TGF- $\beta$ type I receptor into caveolin-1 and EEA1 double-positive early endosomes
}

Kangmin $\mathrm{He}^{1,2}$, Xiaohua $\mathrm{Yan}^{3}$, Nan $\mathrm{Li}^{1}$, Song Dang ${ }^{1}$, Li Xu ${ }^{1}$, Bing Zhao ${ }^{3}$, Zijian $\mathrm{Li}^{2}$, Zhizhen $\mathrm{Lv}^{2}$, Xiaohong Fang ${ }^{1}$, Youyi Zhang ${ }^{2}$, Ye-Guang Chen ${ }^{3}$

${ }^{1}$ Beijing National Laboratory for Molecular Sciences, Key Laboratory of Molecular Nanostructures and Nanotechnology, Institute of Chemistry, Chinese Academy of Sciences, Beijing 100190, China; ${ }^{2}$ Institute of Vascular Medicine Peking University Third Hospital and Academy for Advanced Interdisciplinary Studies, Peking University, Key Laboratory of Cardiovascular Molecular Biology and Regulatory Peptides, Ministry of Health, Key Laboratory of Molecular Cardiovascular Sciences, Ministry of Education and Beijing Key Laboratory of Cardiovascular Receptors Research, Beijing 100191,China; ${ }^{3}$ State Key Laboratory of Biomembrane and Membrane Biotechnology, Tsinghua-Peking Center for Life Sciences, School of Life Sciences, Tsinghua University, Beijing 100084, China

Endocytosis and intracellular sorting of transforming growth factor- $\beta$ (TGF- $\beta$ ) receptors play an important regulatory role in TGF- $\beta$ signaling. Two major endocytic pathways, clathrin- and caveolae-mediated endocytosis, have been reported to independently mediate the internalization of TGF- $\beta$ receptors. In this study, we demonstrate that the clathrin- and caveolae-mediated endocytic pathways can converge during TGF- $\beta$ receptor endocytic trafficking. By tracking the intracellular dynamics of fluorescently-labeled TGF- $\beta$ type I receptor (T $\beta R I)$, we found that after mediating T $\beta R I$ internalization, certain clathrin-coated vesicles and caveolar vesicles are fused underneath the plasma membrane, forming a novel type of caveolin-1 and clathrin double-positive vesicles. Under the regulation of Rab5, the fused vesicles are targeted to early endosomes and thus deliver the internalized T $\beta R I$ to the caveolin-1 and EEA1 double-positive early endosomes (caveolin-1-positive early endosomes). We further showed that the caveolin-1-positive early endosomes are positive for Smad3/SARA, Rab11 and Smad7/Smurf2, and may act as a multifunctional device for TGF- $\beta$ signaling and TGF- $\beta$ receptor recycling and degradation. Therefore, these findings uncover a novel scenario of endocytosis, the direct fusion of clathrin-coated and caveolae vesicles during TGF- $\beta$ receptor endocytic trafficking, which leads to the formation of the multifunctional sorting device, caveolin-1-positive early endosomes, for TGF- $\beta$ receptors.

Keywords: clathrin; caveolae; TGF- $\beta$ receptor; caveolin-1-positive early endosomes; endocytic trafficking Cell Research (2015) 25:738-752. doi: 10.1038/cr.2015.60; published online 22 May 2015

\section{Introduction}

Endocytosis is a key cellular process that is used by eukaryotic cells to internalize a variety of molecules, regulate signal transduction and neurotransmission, and

Correspondence: Xiaohong Fang ${ }^{\mathrm{a}}$, Youyi Zhang ${ }^{\mathrm{b}}$, Ye-Guang Chen ${ }^{\mathrm{c}}$

${ }^{a}$ Tel/Fax: 86-10-6265-3083

E-mail: xfang@iccas.ac.cn

${ }^{\mathrm{b}} \mathrm{Tel} / \mathrm{Fax}: 86-10-6236-1450$

E-mail: zhangyy@bjmu.edu.cn

${ }^{\mathrm{c}}$ Tel: 86-10-6279-5184; Fax: 86-10-6279-4376

E-mail:ygchen@tsinghua.edu.cn

Received 7 May 2014; revised 12 January 2015; accepted 2 February 2015; published online 22 May 2015 modulate the plasma membrane composition [1-3]. Eukaryotic cells have evolved diverse mechanisms of endocytosis, which can be divided into clathrin-dependent and -independent pathways. Clathrin-dependent endocytosis occurs constitutively in all known eukaryotic cells, defined by a requirement for clathrin and adaptor proteins to form the endocytic vesicle coat [2]. One of the most important clathrin-independent pathways is mediated by caveolae, which are flask-shaped plasma membrane invaginations marked by the presence of caveolin-1 [1, 4]. Understanding the mechanism of different endocytic pathways and how these pathways are regulated is of critical importance in the study of cell signaling process, especially for transforming growth factor- $\beta$ (TGF- $\beta$ ) sig- 
naling, where TGF- $\beta$ receptors harness both clathrin- and caveolae-dependent pathways for internalization [5-8].

TGF- $\beta$ and related growth factors regulate a variety of important cellular processes such as cell proliferation, differentiation, motility and apoptosis $[9,10]$. Disregulation of their signal transduction has been associated with several human diseases such as cancer and tissue fibrosis $[11,12]$. TGF- $\beta$ signaling is initiated when TGF- $\beta$ binds to the single transmembrane serine/threonine protein kinase known as TGF- $\beta$ type II receptor (T $\beta$ RII). The TGF- $\beta$ type I receptor (T $\beta R I)$ is then recruited to and activated by TRRII, which in turn leads to the phosphorylation of Smad2/3. The activated Smad2/3-Smad4 complexes are translocated into the nucleus where they bind DNA and other transcriptional machineries to regulate the transcription of target genes $[13,14]$.

Previous studies have shown that TGF- $\beta$ receptors undergo a rapid yet constant internalization in a ligand-dependent or -independent way [5-8, 15-17]. The two major endocytic pathways, clathrin-mediated endocytosis and caveolae-mediated endocytosis, have been reported to independently mediate the internalization of TGF- $\beta$ receptors based on indirect evidence [5-8]. For instance, TGF- $\beta$ receptors were found to associate with clathrin-associated adaptor complex AP-2 or their endocytosis was dampened by potassium depletion, cytosol acidification or hypertonic buffer $[6-8,15,18]$, and TGF- $\beta$ receptors were observed to colocalize with caveolae at the plasma membrane or caveosomes in the cytoplasm [8, $19,20]$. However, up to now, the direct real-time monitoring of TGF- $\beta$ receptor internalization via either caveolae or clathrin has not been achieved yet, which is vital to understand the role of clathrin and caveolae in TGF- $\beta$ receptor endocytosis. Especially, it has been proposed that the internalization of TGF- $\beta$ receptors via the clathrin pathway facilitates the phosphorylation of downstream Smad2/3 in EEA1-positive early endosomes, whereas that the one via caveolae promotes TGF- $\beta$ receptor ubiquitination and degradation in caveosomes [8]. Early endosomes have been recognized as a vital signaling organelle for TGF- $\beta$ signaling $[6,16,21]$. However, in several studies, inhibition of clathrin-mediated endocytosis only slightly affected TGF- $\beta 1$-stimulated TGF- $\beta$ signaling [5, $17,20]$, and disruption of caveolae-mediated endocytosis downregulated TGF- $\beta$ signaling [20], indicating that these two endocytic pathways are not functionally separated. On the other hand, during the infection of certain viruses, it has been observed that the viruses could be exchanged between early endosomes and caveosomes [2225]. However, it is still unclear whether there is a direct interaction between these two endocytic pathways and how the interaction between these two pathways could regulate the endocytic trafficking and signal transduction of TGF- $\beta$ receptors.

In this study, by using an integrative approach of livecell fluorescence microscopy, electron microscopy and biochemical tools, we monitored the internalization of TGF- $\beta$ receptor and discovered the direct fusion of clathrin- and caveolae-mediated endocytic pathways during TGF- $\beta$ receptor endocytic trafficking.

\section{Results}

Real-time observation of caveolae- and clathrin-mediated TGF- $\beta$ receptor endocytosis

We used dual-color total internal reflection fluorescence microscopy (TIRFM) to image cell membrane for the real-time observation of T $\beta R I$ internalization. HeLa cells expressing Myc-tagged T $\beta R I$ (Myc-T $\beta R I)$ were sequentially labeled with anti-Myc antibody and fluorescent-conjugated antibodies as reported [6-8]. Myc-TßRI was expressed at a reasonable level and its overexpression showed minor effect on receptor endocytosis and signal transduction as shown in this study (Supplementary information, Figures S1 and S2) and previous reports [6-8]. To investigate the role of caveolae in T $\beta R I$ internalization, HeLa cells were co-transfected with MycT $\beta R I$ and C-terminally EGFP-tagged caveolin-1 (caveolin-1-EGFP) [26]. Dual-color TIRFM imaging showed that caveolin-1-EGFP and Myc-T $\beta$ RI signals appeared as well-dispersed fluorescent spots at the plasma membrane with partial colocalization $(\sim 13.6 \%$; Figure $1 \mathrm{~A}$ and Supplementary information, Figure S3A). With time-lapse imaging of Myc-TßRI and caveolin-1-EGFP, we noticed the simultaneous fluorescence loss of the colocalized caveolin-1-EGFP and Myc-TßRI spots from the plasma membrane in tens of seconds (Figure 1B and Supplementary information, Movie S1), indicating their co-internalization [27]. Meanwhile, we found that T $\beta R I$ could move toward and then colocalize with the pre-existing caveolae spots at the plasma membrane (Figure 1C and Supplementary information, Movie S2). These results indicate that the pre-existing caveolae at the plasma membrane can mediate T $\beta R I$ endocytosis.

To visualize clathrin-mediated endocytosis, cells were co-transfected with EGFP-clathrin and Myc-T $\beta R I$. Dual-color TIRFM imaging showed that a few TRRI spots were colocalized with clathrin spots at the plasma membrane ( $9.4 \%$; Figure 1D and Supplementary information, Figure S3B). The clathrin spot was found to show up and grow bigger around the pre-existing T $\beta R I$ spot at the plasma membrane. Then the fluorescence of clathrin and T $\beta R I$ disappeared from the plasma membrane simultaneously (Figure 1E and Supplementary information, 

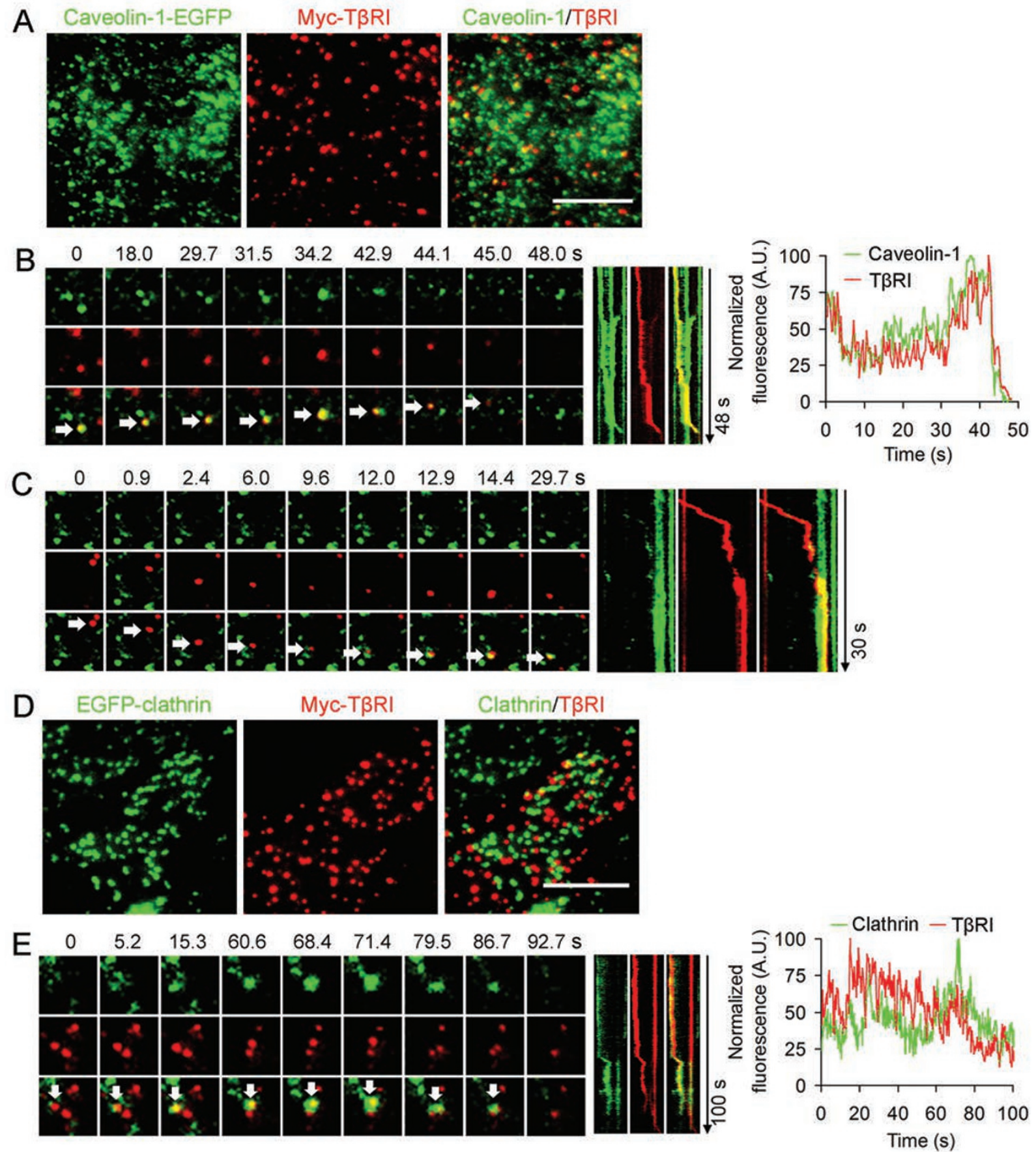

Figure 1 Real-time TIRFM imaging of caveolae- and clathrin-mediated T $\beta R$ I endocytosis. HeLa cells were co-transfected with Myc-T $\beta R$ I and caveolin-1-EGFP (A-C) or EGFP-clathrin (D, E). Myc-T $\beta R I$ was labeled and internalized at $37^{\circ} \mathrm{C}$ in the presence of TGF- $\beta 1$. (A) Dual-color TIRFM images of caveolin-1-EGFP and Myc-T $\beta R I$ at the plasma membrane. (B) Snapshots of a caveolae-mediated Myc-T $\beta R$ I endocytosis event (arrows). Kymographs and fluorescence of the endocytic event over time are shown. (C) Live-cell TIRFM imaging of a Myc-TRRI spot moving toward a pre-existing caveolae spot (arrows). The corresponding kymographs are shown. (D) Dual-color TIRFM images of EGFP-clathrin and Myc-T $\beta$ RI at the plasma membrane. (E) Snapshots of a clathrin-mediated T $\beta$ RI endocytosis event (arrows). Kymographs and fluorescence of the endocytic event over time are shown. Scale bar $=10 \mu \mathrm{m}$. 
Movie S3), indicating clathrin-mediated internalization of T $\beta$ RI.

Intracellular distribution of TGF- $\beta$ receptors in caveolin-1-positive early endosomes

To probe the intracellular distribution of T $\beta R I$, we immunolabeled caveolin-1-EGFP-expressing HeLa cells with anti-T $\beta$ RI (V-22) and anti-EEA1 antibodies and carried out the imaging by confocal microscopy. Unexpectedly, triple-color confocal imaging revealed that the endogenous T $\beta R I$ colocalized with caveolin- 1 and EEA1 double-positive spots (Figure 2A). To further characterize the subcellular distribution of the internalized

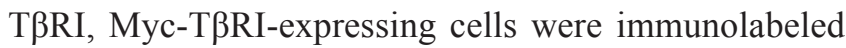
with anti-EEA1 and anti-caveolin-1 antibodies. Consistent with the distribution of endogenous T $\beta R I$, after TGF- $\beta 1$ treatment for $30 \mathrm{~min}$, Myc-T $\beta R I$ signals was distributed in four different intracellular compartments: in addition to the well-known EEA1-positive vesicles, caveolin-1-positive vesicles, EEA1 and caveolin-1 double-negative vesicles, they were also found in EEA1 and caveolin-1 double-positive vesicles (termed the caveolin-1-positive early endosome in this study; $29.9 \%$; Figure $2 \mathrm{~B}$ and $2 \mathrm{C}$, and Supplementary information, Figure S4). In the absence of TGF- $\beta 1$, a similar percentage of T $\beta R I$ signals were found in caveolin-1-positive early endosomes ( $\sim 31.7 \%$; Figure $2 \mathrm{C}$ and Supplementary information, Figure S4E). Upon TGF- $\beta 1$ treatment, the distribution of T $\beta$ RI was increased in EEA1-positive vesicles, but decreased in caveolin-1-positive vesicles (Supplementary information, Figure S4E). Moreover, the distribution of T $\beta R I$ in caveolin-1-positive early endosomes was not cell specific as it was also observed in mink lung epithelial Mv1Lu cells (Supplementary information, Figure S5).

Next, we used an integrative approach of three-dimensional confocal microscopy, triple-color live-cell confocal microscopy, electron microscopy and biochemical tools to confirm the existence of the caveolin-1-positive early endosomes and to further characterize these novel structures: (1) In the $x-z$ and $y-z$ cross-sectional images of the three-dimensional z-stacks, the distribution of TRI in the caveolin-1-positive early endosomes could be readily observed (Figure 2D). (2) As the average size of early endosomes is around the resolution of light microscopy $(\sim 200 \mathrm{~nm})$, we then resolved the ultrastructure of caveolin-1-positive early endosomes by immunoelectron microscopy. By labeling EEA1 and caveolin-1 using different sizes of gold nanoparticles, we found the distribution of caveolin-1 on the membrane of EEA1-positive early endosomes (Supplementary information, Figure S6). (3) We immunoisolated the EEA1-positive early endosomes using a subcellular fractionation protocol [28, 29]. The immunoisolated early endosomes were then analyzed by western blotting with antibodies against EEA1, caveolin-1, TGF- $\beta$ receptor, Golgi protein p115 and mitochondrial protein Tim23. Consistent with the above results, we further confirmed the specific distribution of caveolin-1 and TGF- $\beta$ receptor in EEA1-positive early endosomes, and found that their colocalization was detergent sensitive (Figure 2E). (4) To track caveolin-1-positive early endosomes in live cells, the cells were incubated with fluorescent-conjugated transferrin, a well-characterized marker for early endosomes (Figure 2F). Livecell imaging showed the coordinated movement of MycT $\beta R I$, caveolin-1 and transferrin spots as triple-positive vesicles within cells (Figure 2G and Supplementary information, Movie S4). The fusion and separation of T $\beta$ RI, caveolin- 1 and transferrin molecules were also observed (Figure 2H and Supplementary information, Movie S5). We further examined the intracellular distribution of T $\beta R I$ when the clathrin- or caveolae-mediated endocytosis was interfered (Supplementary information, Figure S7A and S7B). Upon blocking of the clathrin-mediated endocytic pathway by $\mathrm{K}^{+}$depletion [8], the distribution of T $\beta R I$ in EEA1-positive early endosomes was decreased, while its distribution in caveolin-1-positive compartments was increased (Supplementary information, Figure S7C). Upon blocking the caveolae-mediated endocytosis by filipin [20], more TRI molecules were found in the EEA1-positive early endosomes, but less were found in caveolin-1-positive compartments (Supplementary information, Figure S7D). Blocking either clathrin- or caveolae-dependent pathways reduced the distribution of T $\beta R I$ in caveolin-1-positive early endosomes (Supplementary information, Figure S7C and S7D), indicating that both clathrin- and caveolae-mediated endocytosis contribute to the fusion of these two endocytic pathways. Taken together, these results demonstrate that the internalized TGF- $\beta$ receptor could be transported to the novel caveolin-1-positive early endosomes.

\section{Fusion of clathrin-coated vesicles and caveolar vesicles underneath the plasma membrane}

Next, we aimed to investigate where the fusion of the two endocytic pathways is initiated. By imaging the basal plasma membrane of cells co-expressing caveolin-1-EGFP, clathrin-DsRed and Myc-T $\beta R I$, we found that these three molecules showed no obvious colocalization $(\sim 2.6 \%$ of T $\beta$ RI molecules were colocalized with both clathrin and caveolin-1; Figure 3A and Supplementary information, Figure S3C). However, the clathrin and caveolin-1 double-positive vesicles containing T $\beta$ RI were readily observed in the cytoplasm of the same cell $(\sim 27.6 \%$ of 
T $\beta R I$ molecules were colocalized with both clathrin and caveolin-1; Figure 3A). Consistent with this, by immunostaining the cells with antibodies against caveolin-1 and clathrin, T $\beta R I$ signals were also found in clathrin and caveolin-1 double-positive vesicles within cells (Figure 3B).

We further characterized the clathrin and caveolin-1 double-positive vesicles by immunofluorescence, elec-

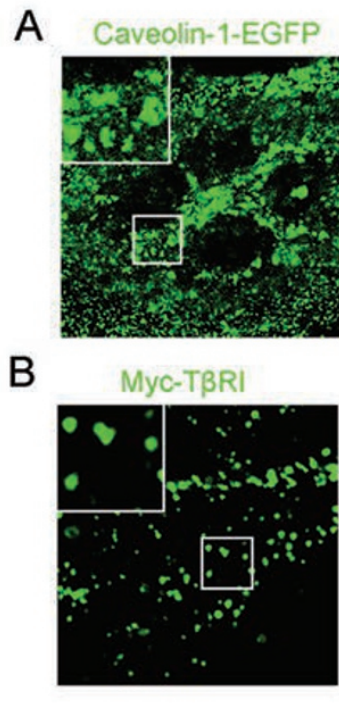

C

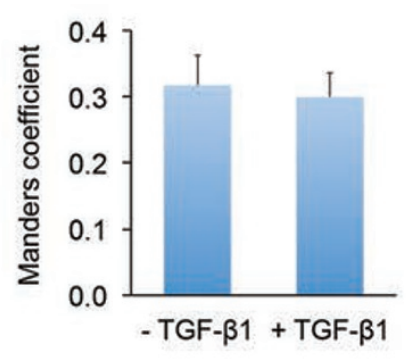

TBRI PAb

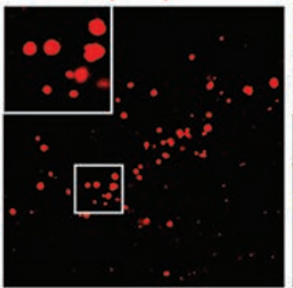

Caveolin-1 pAb

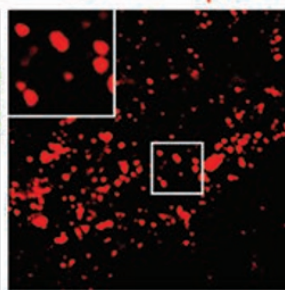

D

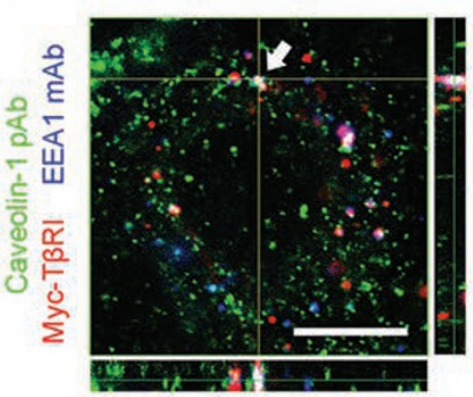

EEA1 mAb

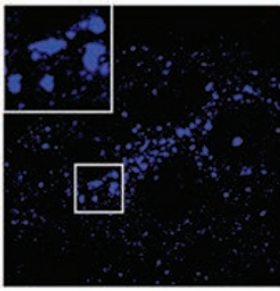

EEA1 mAb

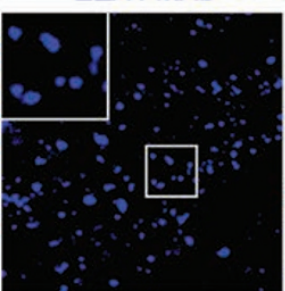

$\%$

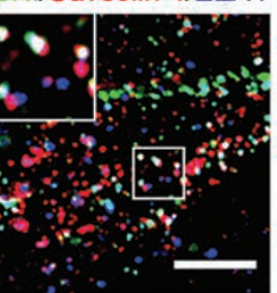

E NP-40 EEA1

Caveolin-1 $T \beta R$ p115

Tim23

F

Caveolin-1-EGFP

Myc-TBRI

Transferrin Caveolin-1/TRRI/Transferrin
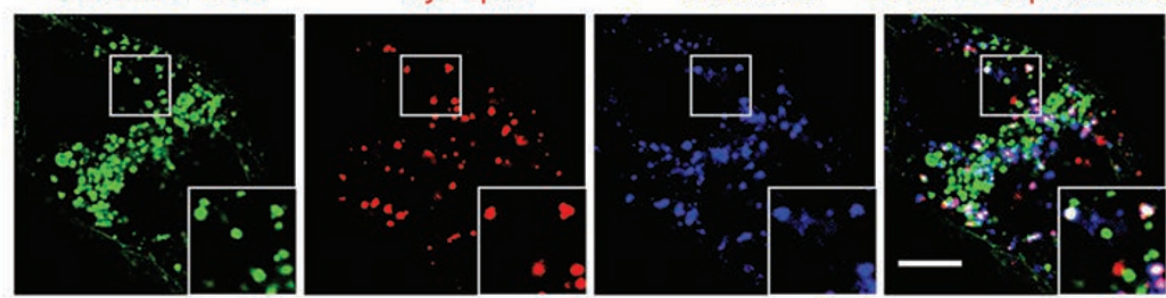

G

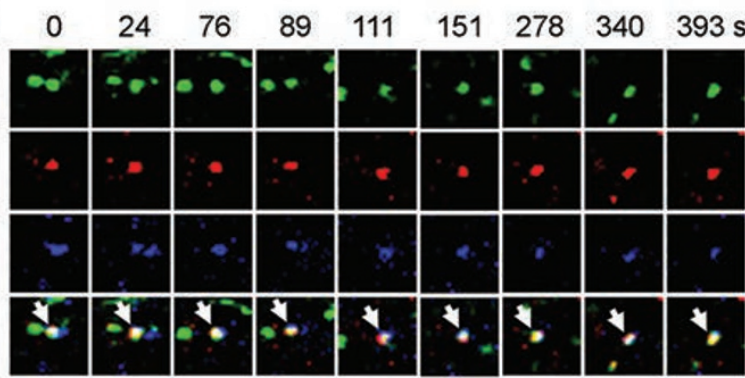

$\mathrm{H}$

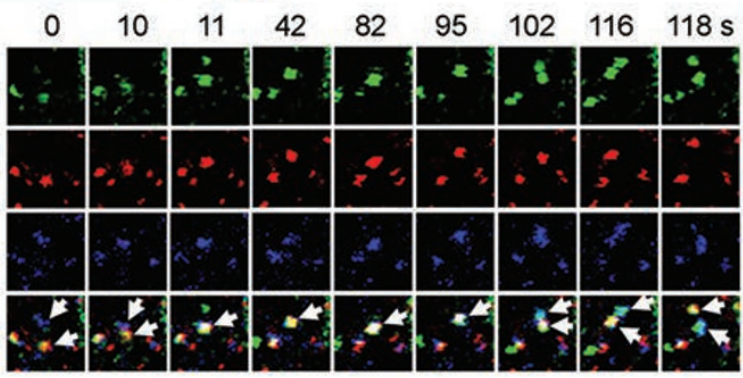


Figure 2 Distribution of T $\beta R I$ in caveolin-1-positive early endosomes. (A) HeLa cells expressing caveolin-1-EGFP were immunostained with antibodies against T $\beta R$ I and EEA1. (B) HeLa cells expressing Myc-T $\beta R I$ were labeled and then incubated at $37^{\circ} \mathrm{C}$ in the presence of TGF- $\beta 1$ to initiate receptor internalization. Thirty minutes later, the cells were immunostained with antibodies against caveolin-1 and EEA1 and imaged by confocal microscopy. (C) Quantification of colocalization of Myc-T $\beta R \mathrm{I}$ with the caveolin-1 and EEA1 double-positive vesicles using the Mander's coefficient (Blobprob plugin, ImageJ). 31.7\% \pm $4.7 \%(n=7$ cells; mean \pm SD) and $29.9 \% \pm 3.7 \%(n=6$ cells $)$ of T $\beta$ RI colocalized with the caveolin-1 and EEA1 double-positive vesicles in the absence and presence of TGF- $\beta 1$, respectively. (D) Cells labeled as in B were imaged by $3 D$ confocal microscopy. A single $z$-plane image and the $x-z$ and $y-z$ cross-sectional images of the cell are shown. (E) The immunoisolated EEA1-positive early endosomes were analyzed by western blotting with antibodies against EEA1, caveolin-1, TGF- $\beta$ receptor, Golgi protein p115 and mitochondrial protein Tim23. (F) Cells expressing caveolin-1-EGFP and Myc-T $\beta$ RI and labeled by Alexa Fluor 633-conjugated transferrin were imaged by confocal microscopy. (G, H) Live-cell confocal imaging showed the intracellular movement $(\mathbf{G})$ and the dynamic fusion and separation $(\mathbf{H})$ of caveolin-1-EGFP, Myc-T $\beta$ RI and tranferrin triple-positive vesicles (arrows). pAb, polyclonal antibody; mAb, monoclonal antibody. Scale bars, $10 \mu \mathrm{m}$ (A, D, F), $5 \mu \mathrm{m}$ (B).

tron microscopy and triple-color live-cell imaging. (1) By immunostaining endogenous caveolin-1 and clathrin in cells without Myc-T $\beta$ RI expression, we found that clathrin- and caveolin-1-labeled vesicles were partially overlapped (Figure 3C). Further analysis revealed that centers of clathrin-coated and caveolar vesicles were $\sim 130 \mathrm{~nm}$ apart (Figure 3C). (2) By using transmission electron microscopy, we observed morphologically characteristic clathrin-coated pits and caveolae (the flaskshaped membrane invagination) at the plasma membrane as previously described $[30,31]$. The partially fused caveolar and clathrin-coated vesicles were also observed (Figure 3D). (3) By live-cell imaging, we revealed the coordinated movement of the fluorescent protein-labeled caveolin-1 and clathrin from the lateral plasma membrane to the cytoplasm (Figure 3E and Supplementary information, Movie S6). The caveolin-1, clathrin and T $\beta$ RI triple-positive vesicles were also found to move from the lateral plasma membrane to the cytoplasm (Figure 3F and Supplementary information, Movie S7). Thus, the above results revealed that the internalized clathrin-coated vesicles and caveolar vesicles could be fused to form caveolin-1 and clathrin double-positive organelle underneath the plasma membrane.

We also examined this fused pathway with another growth factor receptor, the receptor tyrosine kinase epidermal growth factor receptor (EGFR). EGFR has been shown to utilize clathrin- but not caveolae-mediated pathway to enter cells after activation [32]. Consistent with previous studies [32], we found that the activated EGFR was colocalized extensively with clathrin but not caveolin-1 at the plasma membrane by dual-color TIRFM (Supplementary information, Figure S8A and S8B). However, the internalized EGFR molecules were distributed in clathrin and caveolin-1 double-positive vesicles, and then were transported to caveolin-1-positive early endosomes as well (Supplementary information, Figure S8C-S8E). These results suggest that not only the serine/ threonine kinase TGF- $\beta$ receptor but also the receptor tyrosine kinase EGFR can utilize the fusion pathway for endocytic trafficking.

Rab5 regulates the formation of caveolin-1-positive early endosomes from caveolin-1 and clathrin double-positive vesicles

The small GTPase Rab5 is a crucial regulator in the early stages of endocytic trafficking by modulating the fusion of clathrin-coated vesicles with early endosomes $[33,34]$. Studies also found that Rab5 could directly bind to the transmembrane scaffold and C-terminal domains of caveolin-1 [35]. We aimed to study whether Rab5 regulates the fusion of the clathrin-coated vesicles with caveolar vesicles, or the fused vesicles with early endosomes. In cells co-expressing ECFP-Rab5, caveolin-1-EGFP, clathrin-DsRed and Myc-T $\beta R I$, intracellular colocalization of these four molecules was readily observed by multi-color imaging (Figure 4A). We also observed the constant movement of caveolin-1-EGFP, mRFP-Rab5 and Myc-TßRI triple-positive vesicles within cells by live-cell imaging (Figure 4B and Supplementary information, Movie S8), indicating the spatial association among caveolin-1, Rab5 and T $\beta$ RI. In cell expressing the dominant-negative Rab5 mutant (Rab5S34N), the number of EEA1-positive early endosomes was significantly reduced. However, the caveolin-1 and clathrin double-positive vesicles were unaffected (Figure 4C). In addition, we found that the caveolin-1 and clathrin double-positive vesicles were negatively stained with the endosomal SNARE (Soluble N-ethylmaleimidesensitive factor attachment protein receptors) proteins Vtila and syntaxin 6 (Supplementary information, Figure S9). Thus, these results suggest that the fusion of clathrin-coated and caveolar vesicles occurs ahead of their fusion with early endosomes.

To further determine the role of Rab5 in the formation of caveolin-1-positive early endosomes and the intracel- 
lular distribution of T $\beta R I$, we transfected Myc-T $\beta R I-e x-$ pressing cells with wild-type Rab5, inactive mutant Rab5S34N or constitutively active mutant Rab5Q79L. In cells expressing wild-type Rab5, 53.1\% of T $\beta$ RI signals were observed in caveolin-1-positive early endosomes 30 min post internalization (Figure 4D and 4G). Rab5S34N expression substantially inhibited the colocalization of T $\beta R I$ with caveolin-1-positive early endosomes (10.7\%;

A

Caveolin-1-EGFP Clathrin-DsRed Myc-T $\beta R I$
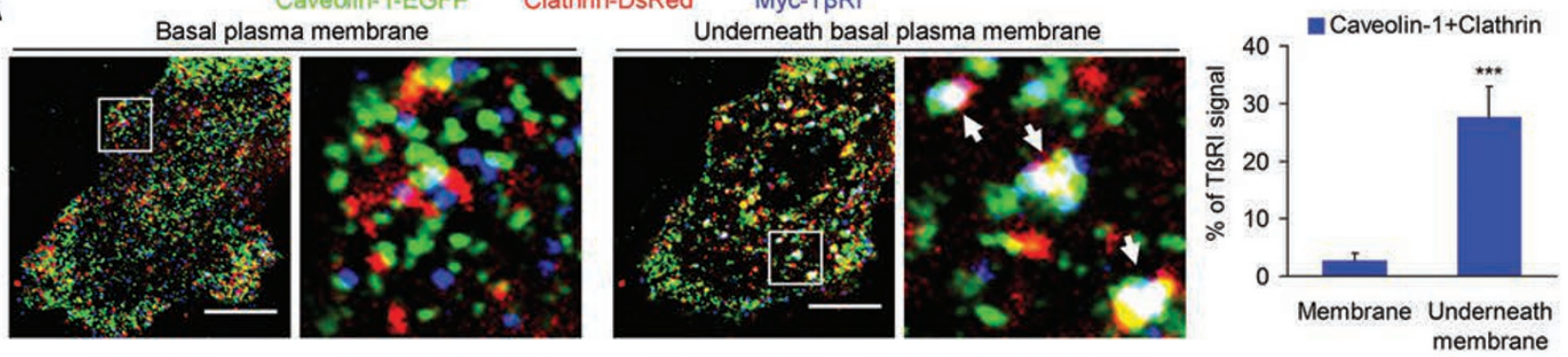

B

Myc-T $\beta R$ I

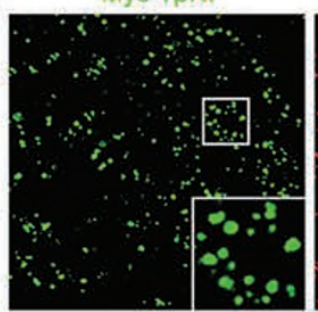

Caveolin-1 PAb

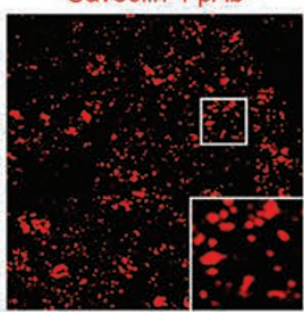

Clathrin $\mathrm{mAb}$
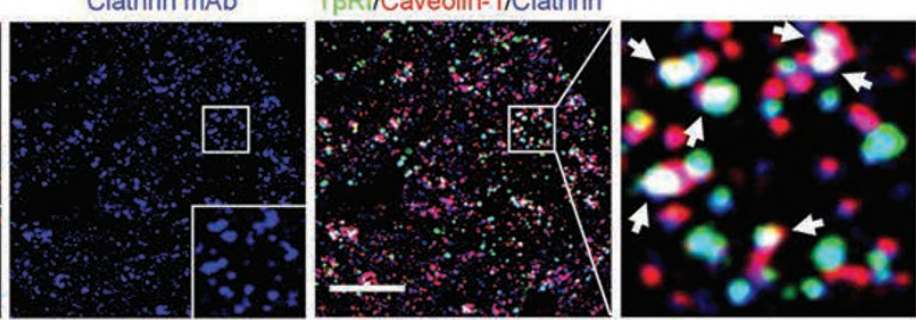

C

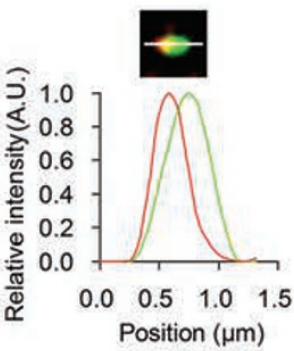

D

Clathrin-coated vesicles Caveolae
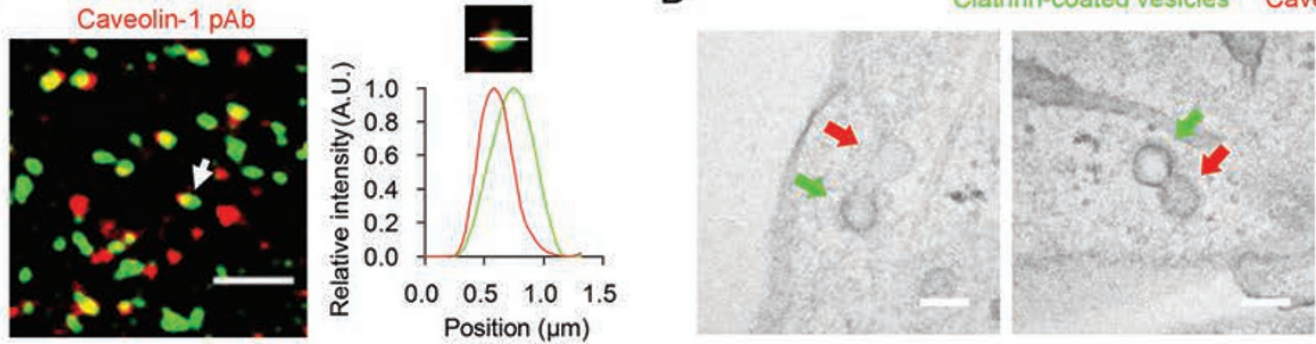

E

Caveolin-1-EGFP
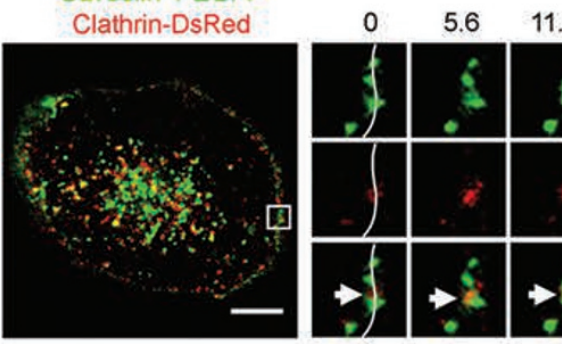

20.8

29.0

38.8
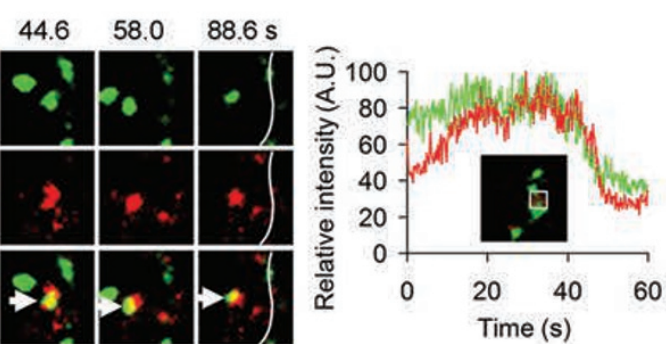

F

Caveolin-1-EGFP
Clathrin-DsRed Myc-T $\beta R I$
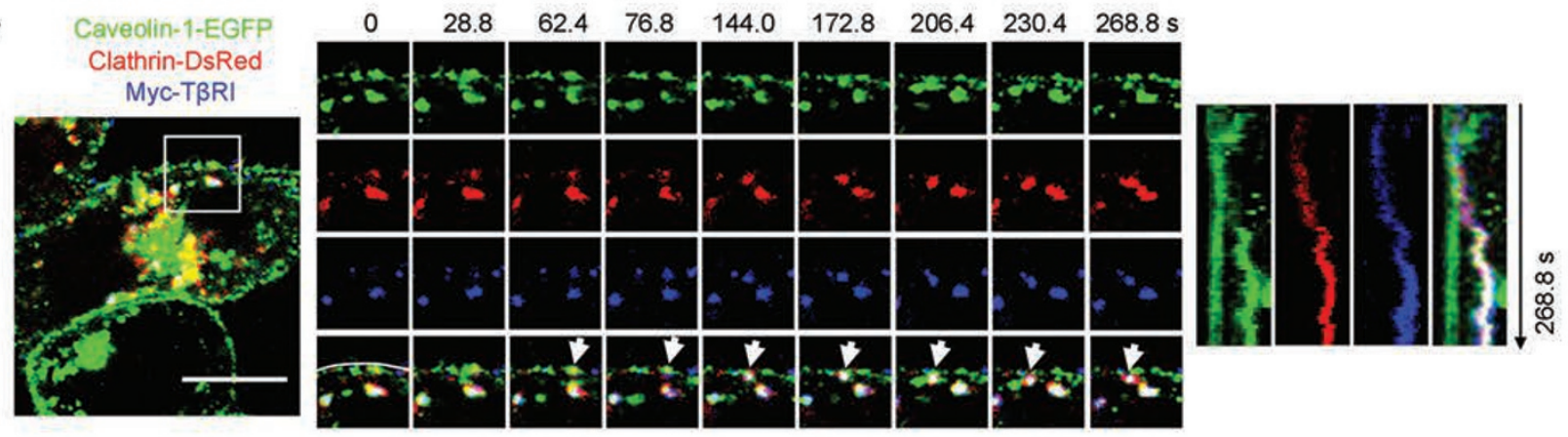
Figure 3 Fusion of clathrin-coated vesicles and caveolar vesicles underneath the plasma membrane. (A) The HeLa cell co-expressing caveolin-1-EGFP, clathrin-DsRed and Myc-T $\beta$ RI was imaged at the basal plasma membrane plane or underneath the basal plasma membrane (the plane $1 \mu \mathrm{m}$ above the basal plasma membrane) by confocal microscopy. The distribution of T $\beta \mathrm{RI}$ in caveolin-1 and clathrin double-positive vesicles was quantified (mean $\pm \mathrm{SD}, n=5$ cells, ${ }^{* * *} P<0.001$, Student's t-test). (B) Myc-T $\beta$ RI-expressing cells were immunostained with antibodies against caveolin-1 and clathrin and imaged by confocal microscopy. Myc-T $\beta$ RI was labeled and then internalized for $15 \mathrm{~min}$ at $37^{\circ} \mathrm{C}$ in the presence of TGF- $\beta 1$. The triple-positive vesicles were marked by arrows in A and B. (C) HeLa cells were immunostained with antibodies against caveolin-1 and clathrin and imaged by confocal microscopy. The arrow-pointed vesicle is enlarged, rotated and shown on the right. The fluorescence of clathrin and caveolin-1 along the white line on the vesicle is shown. (D) Imaging the ultrastructure of the partially fused caveolar and clathrin-coated vesicles within cells by electron microscopy (green arrow, clathrin-coated vesicle; red arrow, caveolin-1-positive vesicle). (E) Live-cell spinning-disk confocal imaging of the middle plane of the cells co-expressing caveolin-1-EGFP and clathrin-DsRed. The boxed region was magnified (images to the right) to show the movement of a caveolin-1-EGFP and clathrin-DsRed double-positive vesicle (arrows) from the lateral plasma membrane (white lines) to the cytoplasm. The corresponding fluorescence time traces of the inset boxed region are shown. (F) Triple-color live-cell confocal imaging of the cell co-expressing caveolin-1-EGFP, clathrin-DsRed and Myc-T $\beta$ RI. The boxed region was magnified (images to the right) to show the movement of a Myc-T $\beta R I$, caveolin-1-EGFP and clathrin-DsRed triple-positive vesicle (arrows) from the lateral plasma membrane (white lines) to the cytoplasm. The corresponding kymographs of these three molecules are shown. Scale bars, $10 \mu \mathrm{m}$ (A, B, E, F), $2 \mu \mathrm{m}$ (C), $100 \mathrm{~nm}$ (D).

Figure 4E and 4G). Remarkably, Rab5Q79L expression (with a similar expression level to wild-type Rab5 and Rab5S34N) resulted in enlarged caveolin-1-positive early endosomes where $81.2 \%$ of T $\beta$ RI signals were trapped (Figure $4 \mathrm{~F}$ and $4 \mathrm{G}$ ). These results suggest that Rab5 regulates the formation of caveolin-1-positive early endosomes and the intracellular distribution of T $\beta$ RI.

Detection of SARA, Rab11 and Smad7/Smurf2 in caveolin-1-positive early endosomes

The distribution of T $\beta R I$ in caveolin-1-positive early endosomes raises the question about the role of caveolin-1-positive early endosomes in TGF- $\beta$ signaling. Early endosomes have been recognized as an important signaling center for TGF- $\beta /$ Smad signaling $[6,8,36]$. In cells co-expressing EGFP-Smad3, caveolin-1-mRFP and Rab5Q79L, and immunostained for EEA1, we observed the accumulation of EGFP-Smad3 in the enlarged caveolin-1-positive early endosomes (Figure 5A). SARA (Smad anchor for receptor activation), a FYVE domain-containing protein, has been reported to facilitate $\mathrm{Smad} 2 / 3$ phosphorylation by TGF- $\beta$ receptor in early endosomes $[6$, 37]. By immunostaining for SARA, we found that SARA was also located in caveolin-1-positive early endosomes (Figure 5B), indicating that caveolin-1-positive early endosomes may be involved in TGF- $\beta$ signaling.

We also observed a sudden appearance of overlapped caveolin-1-EGFP and Myc-T $\beta$ RI spots at the plasma membrane by TIRFM imaging several minutes after the initiation of T $\beta R I$ internalization (Supplementary information, Figure S10A). In addition, the caveolin-1-EGFP and Myc-T $\beta R I$ double-positive vesicles were observed to move actively toward the plasma membrane from the cell interior (Supplementary information, Figure S10B).
These results suggest that the caveolin-1-containing vesicles are involved in T $\beta$ RI recycling. To confirm this, we examined whether Rab11, a small GTPase regulating T $\beta$ RI recycling [7], is associated with the caveolin-1-containing vesicles. In cells co-expressing ECFPRab11, caveolin-1-EGFP and Myc-TßRI and labeled for transferrin, the vesicles positive for these four molecules were observed (Figure 5C). The association of Rab11, caveolin-1 and T $\beta$ RI was further confirmed by their coordinated movement in a live cell (Figure 5D and Supplementary information, Movie S9). As the caveolae/ raft components have been reported to be enriched in recycling endosomes to regulate protein/lipid sorting and trafficking [38], these results suggest that caveolin-1-positive early endosomes may be involved in Rab11-regulated T $\beta$ RI recycling.

Caveolae-mediated endocytosis has been shown to negatively regulate TGF- $\beta$ signaling by promoting TGF- $\beta$ receptor turnover, probably via Smad7/Smurf-catalyzed ubiquitination $[8,39]$. The existence of caveolin-1 in early endosomes led us to ask whether caveolin-1-positive early endosomes participate in T $\beta R I$ degradation. In cells co-expressing caveolin-1-ECFP, Myc-T $\beta R I$ and Smad7-HA and immunostained for EEA1, the colocalization of these four molecules was observed in the cytoplasm (Figure 5E). Similarly, Smurf2 was also found to associate with the caveolin-1-positive early endosomes (Figure 5F). These results suggest that by recruiting Smad7/Smurf2, the caveolin-1-positive early endosomes may be involved in T $\beta R I$ degradation.

\section{Discussion}

During the infection of viruses such as Simian Virus 

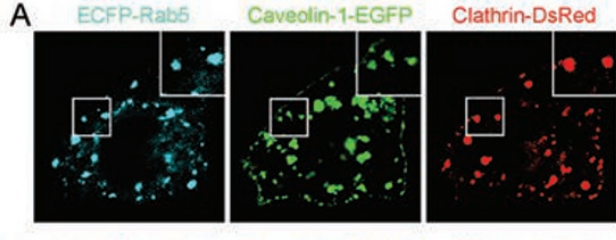

Myc-TßRI Caveolin-1/Clathrin/TßRI

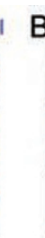

C ECFP-Rab5S34N Caveolin-1-EGFP

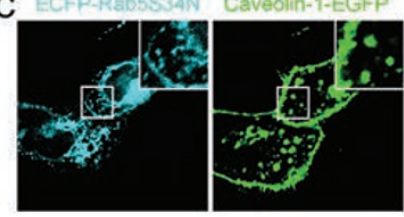

Clathrin-DsRed
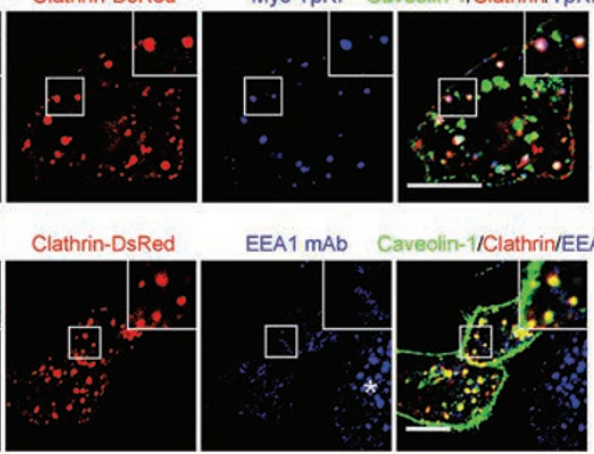

EEA1 $\mathrm{mAb}$
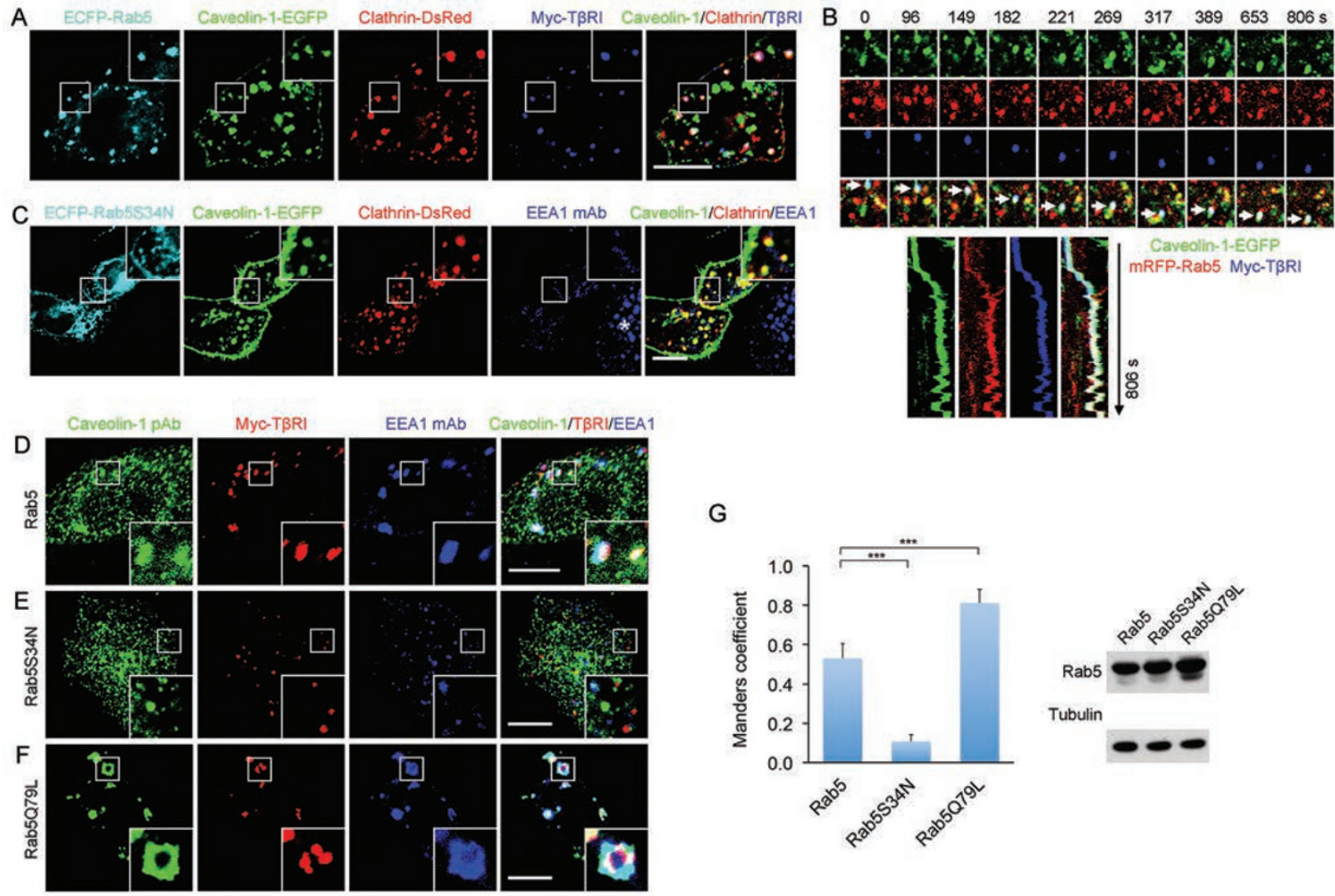

G
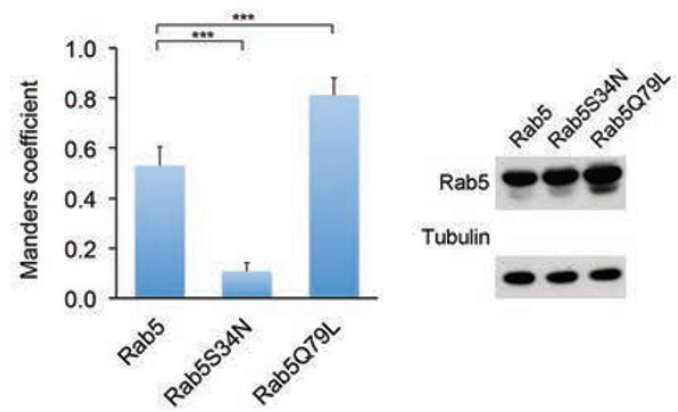

Tubulin

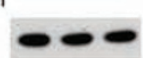

Figure 4 Rab5 regulates the formation of caveolin-1-positive early endosomes. (A) Confocal images of HeLa cells co-expressing ECFP-Rab5, caveolin-1-EGFP, clathrin-DsRed and Myc-T $\beta$ RI. Myc-T $\beta$ RI was labeled and then internalized for 15 min in the presence of TGF- $\beta 1$. The caveolin-1-EGFP, clathrin-DsRed and Myc-T $\beta R I$ images were overlaid and shown on the right. (B) Live-cell confocal imaging revealed the intracellular movement of a caveolin-1-EGFP, mRFP-Rab5 and Myc-T $\beta$ RI triple-positive vesicle (arrows). The corresponding kymographs of these three molecules are shown. (C) Confocal images of cells co-expressing ECFP-Rab5S34N, caveolin-1-EGFP and clathrin-DsRed and immunostained for EEA1. The caveolin-1-EGFP, clathrin-DsRed and EEA1 images were overlaid and shown on the right. Rab5S34N overexpression reduced the number of EEA1-labeled early endosomes compared with the non-transfected cells (marked by the star). (D-F) HeLa cells were co-transfected with Myc-TßRI and ECFP-Rab5 (D), ECFP-Rab5S34N (E) or Rab5Q79L (F). Myc-T $\beta$ RI was labeled and then internalized for $30 \mathrm{~min}$ in the presence of TGF- $\beta 1$. Then the cells were immunostained with antibodies against caveolin-1 and EEA1 and imaged by confocal microscopy. (G) Quantification of the colocalization of Myc-T $\beta R I$ with caveolin-1 and EEA1 in D-F using the Mander's coefficient (mean $\pm \mathrm{SD}, n=7-10$ cells/condition). The expression levels of wild-type and mutant Rab5 were examined by western blotting. ${ }^{* * *} P<0.001$ (ANOVA). Scale bar $=10 \mu \mathrm{m}$.

40, bovine papillomavirus type 1 and John Cunningham virus, they were found to locate in the caveolin-1 and EEA1 double-positive vesicles within cells [22-25]. As these viruses are internalized via either caveolaeor clathrin-mediated endocytosis, it has been proposed that caveolin-1 and EEA1 double-positive vesicles are originated from either the direct transport of the initially internalized caveosomes to early endosomes $[23,40]$ or the sorting of clathrin/early endosomal vesicles to the caveolin-1-positive compartments [24]. However, there is no direct evidence on whether and how the convergence of these two pathways occurs. By tracking the intracellular dynamic trafficking of T $\beta R I$, we observed the direct fusion of clathrin-coated vesicles and caveolar vesicles upon their internalization, which occurs before they reach the early endosome and contribute to the formation of the multifunctional sorting device, caveolin-1-positive early endosomes, for TGF- $\beta$ receptors (Figure 6).

When clathrin-coated pits are pinched off from the plasma membrane, the free clathrin-coated vesicles rap- 

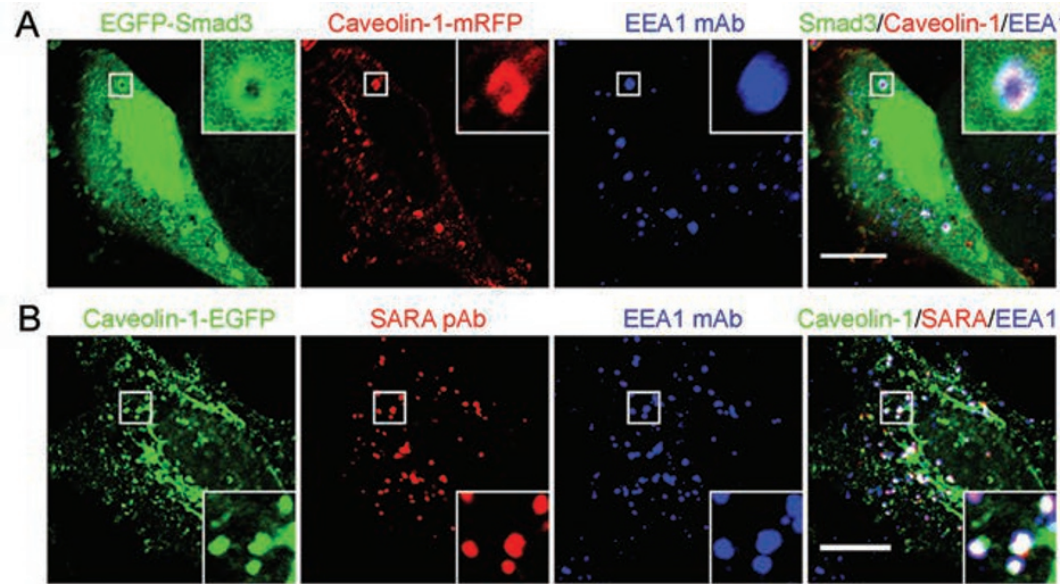

SARA PAb

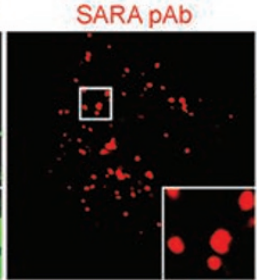

EEA1 mAb
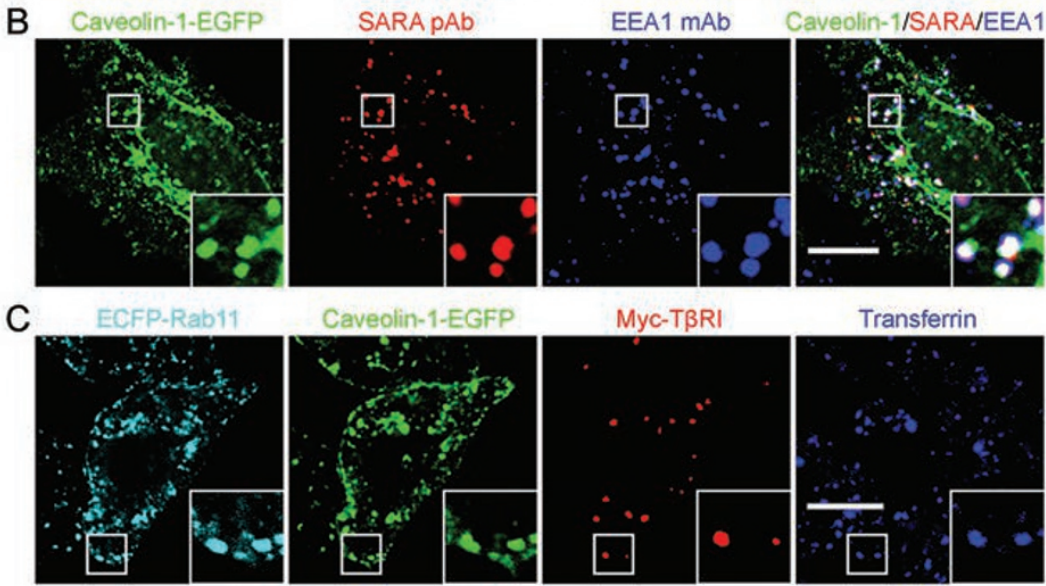

Caveolin-1-EGFP
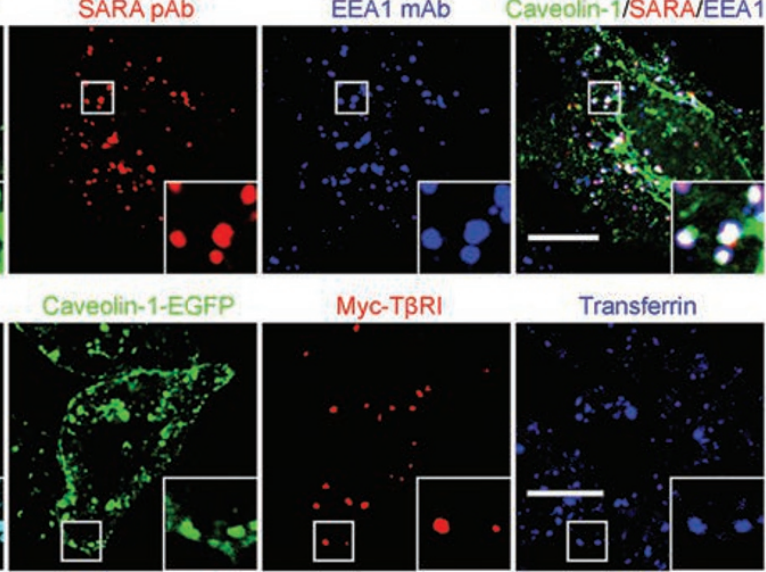

Myc-TßRI

Transferrin
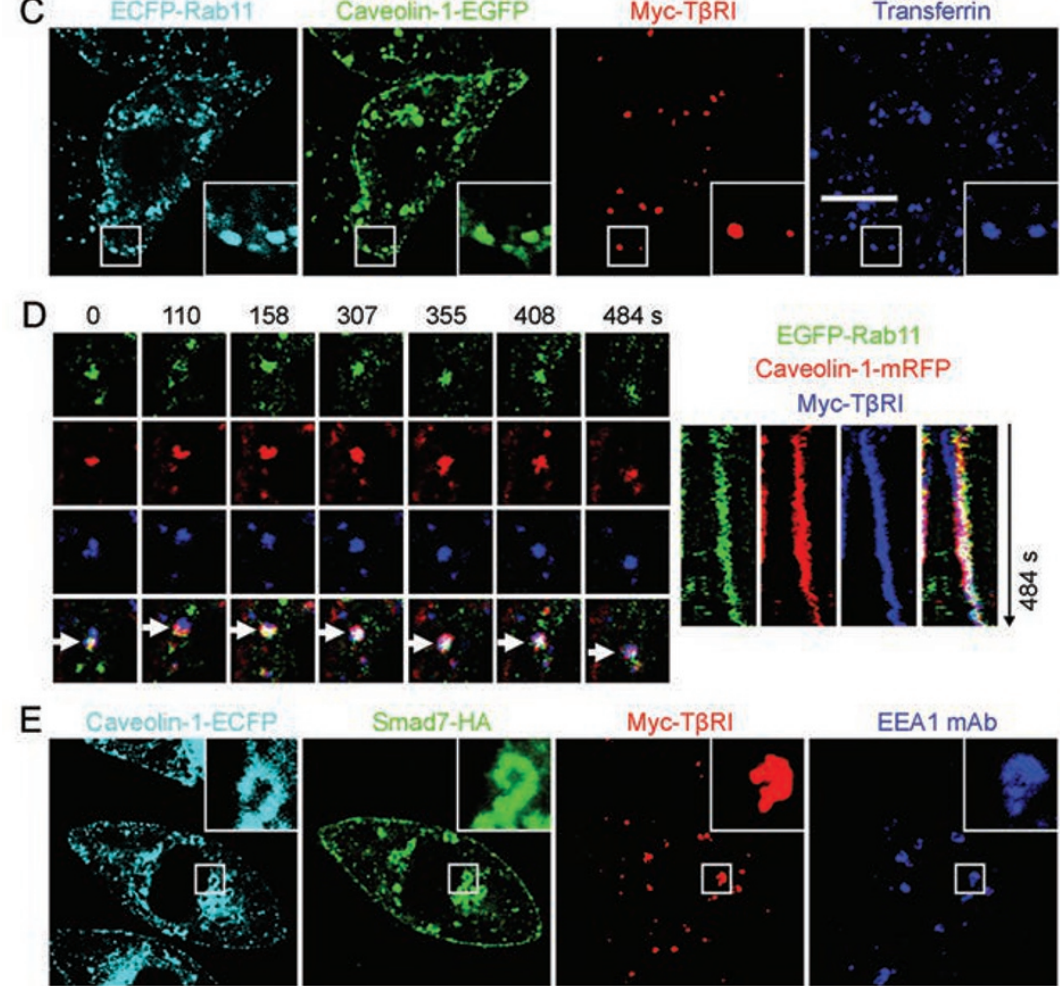

Myc-T $\beta R \mid$
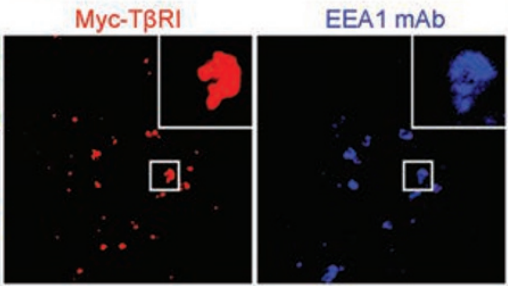

Smad7/TRRI/EEA1

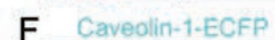

Smurf2-Flag

Myc-T $\beta R I$
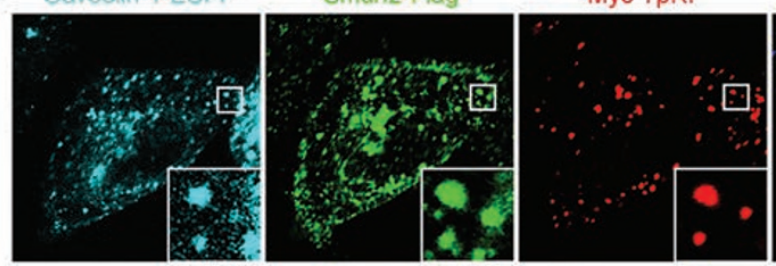

EEA1 mAb

Smur2/TßRI/EEA1
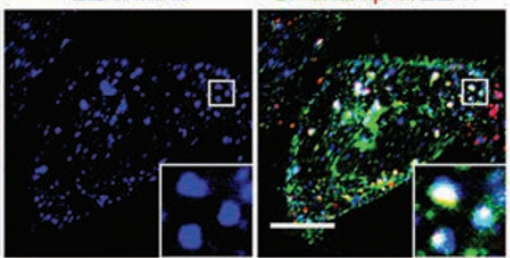

Figure 5 Detection of Smad3/SARA, Rab11 and Smad7/Smurf2 in caveolin-1-positive early endosomes. (A) Confocal images of HeLa cells co-expressing Rab5Q79L, EGFP-Smad3 and caveolin-1-mRFP and immunostained for EEA1. (B) Confocal images of cells co-expressing caveolin-1-EGFP and SARA and immunostained for EEA1 and SARA. (C) Confocal images of cells co-expressing ECFP-Rab11, caveolin-1-EGFP and Myc-T $\beta$ RI and labeled by Alexa Fluor 633-conjugated transferrin. (D) Live-cell confocal imaging revealed the intracellular movement of an EGFP-Rab11, caveolin-1-mRFP and Myc-T $\beta R I$ triple-positive vesicle (arrows). The corresponding kymographs are shown. (E, F) Confocal images of cells co-expressing caveolin-1-ECFP, Myc-T $\beta$ RI and Smad7-HA (E) or Smurf2-Flag (F). Myc-T $\beta$ RI was labeled and internalized for 30 min in the presence of TGF- $\beta 1$. Then the cells were immunostained with antibodies against EEA1 and HA or Flag. The Myc-T $\beta R I$ and EEA1 images together with the Smad7-HA or Smurf2-Flag images were overlaid and shown on the right. Scale bar $=10 \mu \mathrm{m}$. 


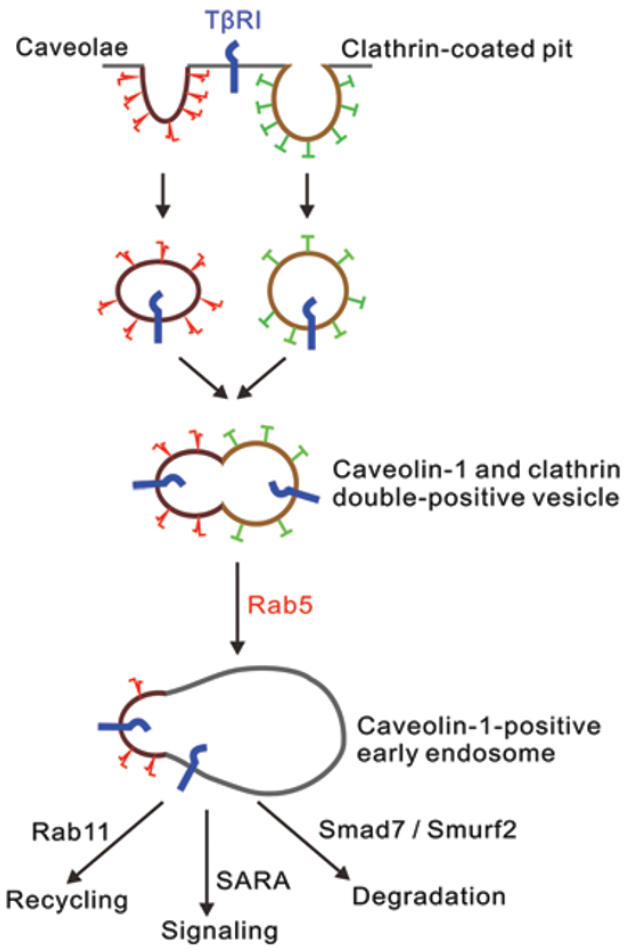

Figure 6 Schematic representation of the convergence of clathrin- and caveolae-mediated endocytic pathways during T $\beta R I$ endocytic trafficking. Although clathrin-coated pits and caveolae can mediate the internalization of $T \beta R \mathrm{R}$ at the plasma membrane independently, a portion of clathrin-coated vesicles and caveolar vesicles are fused after their internalization into cells. Under the regulation of Rab5, the fused vesicles are further targeted to early endosomes to deliver the internalized T $\beta$ RI $(\sim 30 \%)$ to the caveolin-1-positive early endosomes. As a multi-component organelle, the caveolin-1-positive early endosome could act as a multifunctional sorting center for T $\beta R \mathrm{R}$.

idly lose their clathrin coats through a process mediated by Hsc70 and auxilin [41-43]. Still, clathrin-coated vesicles can be frequently observed underneath the plasma membrane as shown in this study and previous reports $[44,45]$. Although it is well recognized that caveolae and clathrin-coated pits are separately located invaginations at the plasma membrane $[2,30]$, the distribution of clathrin-coated vesicles and caveolar vesicles within the cell is less understood. By electron microscopy, we revealed that certain clathrin-coated vesicles and caveolar vesicles were partially fused. When the partially coated clathrin structures (e.g., clathrin plaques and the clathrin-coated vesicles undergoing coat disassembly) expose the uncovered lipid membranes to the adjacent caveolar vesicles [46], the two vesicles could undergo partial fusion. Membrane fusion is usually mediated by Rab and SNARE proteins [47]. Our results revealed that the SNARE pro- teins (Vtila and syntaxin 6) were not associated with the fused clathrin and caveolin-1 double-positive vesicles. Moreover, although Rab5 was located in the fused caveolar and clathrin-coated vesicles, the dominant-negative mutant of Rab5 did not impair the fusion of caveolar vesicles with clathrin-coated vesicles, but reduced the formation of caveolin-1 and EEA1 double-positive vesicles. Therefore, by targeting the caveolar and clathrin-coated vesicles, Rab5 could guide the caveolar and clathrin-coated vesicles to early endosomes, which leads to the formation of the multifunctional cavelin-1-positive early endosomes.

It is known that the distinct endosomal membrane domains contain different compositions, such as Rab4, Rab5, Rab7, Rab11, Arf1, COPI, caveolin-1, clathrin and SARA, either by transient interaction or stable association $[8,23,48]$. These sub-domains endow early endosomes with variable functions, such as sorting cargoes to recycling or degradation compartments, accelerating virus infection and enhancing signaling transduction $[8$, 23-25, 48]. In this study, we demonstrate the existence of the multi-component early endosomes containing EEA1, caveolin-1, Rab5, TßRI, Smad3/SARA, Smad7/Smurf2 and Rab11. The multi-component and multi-functional organelle provides a favorable place for TGF- $\beta$ receptors to access these different molecules easily, thus efficiently promoting TGF- $\beta$ signaling and T $\beta$ RI recycling and degradation. However, the exact location and dynamics of each protein in the membrane of early endosomes is still unknown, and the nano-scale live-cell imaging by multi-color super-resolution microscopy may help solve these issues. Meanwhile, whether the caveolin-1-positive early endosome is a multifunctional organelle for the other internalized receptors needs further investigation.

Therefore, although extracellular cargoes such as TGF- $\beta$ receptors can choose either clathrin- or cavelae-mediated endocytic pathways for internalization at the plasma membrane, it is possible that they are also delivered to a fusion pathway in the cytoplasm to receive a second run of sorting.

\section{Materials and Methods}

\section{Cell culture}

HeLa and T $\beta$ RI-deficient mink lung epithelial L17 cells $[5,49]$ were cultured in Dulbecco's modified Eagle's medium (DMEM, Gibco) supplemented with 10\% fetal bovine serum (Hyclone) and antibiotics (50 mg/ml streptomycin, $50 \mathrm{U} / \mathrm{ml}$ penicillin) at $37{ }^{\circ} \mathrm{C}$ in a $5 \% \mathrm{CO}_{2}$ atmosphere.

\section{Plasmids and transfection}

Plasmids of Myc-T $\beta$ RI and Smad7-HA were constructed as previously described $[50,51]$. Full-length human Smad3 cDNA 
was ligated into the pEGFP-C1 construct (Clonetech) to generate the EGFP-Smad3 plasmid. Plasmids encoding clathrin-DsRed, caveolin-1-EGFP, EGFP-clathrin, EGFP-Rab5 and Rab5Q79L were generous gifts from Drs Sanford M Simon [52], Richard D Minshall [26], Lois E Greene [53], Yan Chen [54] and Harald Stenmark [55], respectively. Plasmids encoding ECFP-Rab5, ECFPRab5S34N, EGFP-Rab11 and ECFP-Rab11 were generous gifts from Marino Zerial [56]. Plasmids encoding mRFP-Rab5 [48] and caveolin-1-mRFP [57] were purchased from Addgene (Addgene plasmids 14437 and 14434, respectively). Transfection was performed using Lipofectamine 2000 (Invitrogen) according to the manufacturer's instructions.

\section{Antibodies and reagents}

Antibodies against c-Myc (9E10, 1:200), HA (Y-11, 1:100), T $\beta R I$ (V-22, 1:100), SARA (1:100), P-Smad2/3 (1:100) and clathrin (1:100) were from Santa Cruz Biotechnologies. Anti-Flag M2 antibody was from Sigma-Aldrich (1:500). Antibodies against caveolin-1 (1:100) and EEA1 (1:200) were from BD Biosciences. Mouse, Rat and/or rabbit Alexa Fluor 488-, 555- or 633-conjugated secondary antibodies (1:500), Alexa Fluor 633-conjugated transferrin $(20 \mu \mathrm{g} / \mathrm{ml})$ and EGF-rhodamine $(80 \mathrm{ng} / \mathrm{ml})$ were all from Molecular Probes. Human TGF- $\beta 1$ was from R\&D (10 ng/ $\mathrm{ml})$. Filipin was obtained from Sigma-Aldrich. The $\mathrm{K}^{+}$-depletion media $\left(140 \mathrm{mM} \mathrm{NaCl}, 1 \mathrm{mM} \mathrm{CaCl}, 1 \mathrm{mM} \mathrm{MgSO}_{4}, 5.5 \mathrm{mM}\right.$ glucose, $0.5 \%$ BSA, and $20 \mathrm{mM}$ HEPES, pH 7.5) and control media (the $\mathrm{K}^{+}$-depletion media supplemented with $10 \mathrm{mM} \mathrm{KCl}$ ) were prepared as previously described [8].

\section{Immunofluorescence microscopy}

Cells were permeabilized in PBS containing 0.5\% Triton X-100 for $10 \mathrm{~min}$, and fixed with $4 \%$ formaldehyde in PBS for $20 \mathrm{~min}$ at room temperature. Then the cells were washed with PBS for three times and incubated in the blocking buffer (PBS, $0.5 \%$ bovine serum albumin) for $30 \mathrm{~min}$. After that, the cells were incubated with the diluted primary antibodies in the blocking buffer at $4{ }^{\circ} \mathrm{C}$ overnight, washed with PBS for three times, and then incubated with the diluted fluorescent-conjugated secondary antibodies in blocking buffer at $4{ }^{\circ} \mathrm{C}$ for $1 \mathrm{~h}$. For labeling with two or more antibodies, the procedures were repeated.

The prepared cell samples were imaged with a confocal microscope (FluoView FV1000-IX81, Olympus, Japan) equipped with a $100 \times / 1.40$ NA objective. For multi-color imaging, ECFP was excited by a $405 \mathrm{~nm}$ laser (FV5-LD405-2, Olympus) and the emission was collected using a $425-475 \mathrm{~nm}$ bandpass filter. EGFP and Alexa Fluor 488 were excited by a $488 \mathrm{~nm}$ laser (FV5-LAMAR, Olympus) and the emission was collected using a 500-545 nm bandpass filter. DsRed, mRFP, Alexa Fluor 555 and Rhodamine were excited by a $559 \mathrm{~nm}$ laser (FV10-LD559, Olympus) and the emission was collected using a 570-625 nm bandpass filter. Alexa Fluor 633 was excited by a $635 \mathrm{~nm}$ laser (FV10-LD635, Olympus) and the emission was collected using a 655-755 nm bandpass filter. For multi-color imaging, the fluorophores were excited by the corresponding lasers and the fluorescence signals were collected by the corresponding emission filters sequentially. In this setup and under our experiment condition, the color bleed-through between different channels is negligible (Supplementary information, Figure S11).

To image the subcellular distribution of Myc-TßRI at the basal plasma membrane plane and underneath the basal plasma membrane of the same cell, the objective was firstly focused on the basal plasma membrane and one image was collected. Then the objective was moved up for $\sim 1 \mu \mathrm{m}$ driven by the Z-piezo to focus on a new plane within the cell but still near the basal plasma membrane (underneath the basal plasma membrane) and another image was collected.

\section{Live-cell fluorescence imaging}

Live-cell receptor labeling was carried out as previously described [8]. Briefly, cells expressing Myc-T $\beta R I$ were sequentially incubated with anti-Myc antibody and the fluorescent-conjugated secondary antibody at $4{ }^{\circ} \mathrm{C}$ for $1 \mathrm{~h}$. Then the cells were transferred to $37^{\circ} \mathrm{C}$ to initiate receptor internalization.

Dual-color TIRFM was performed using a highly sensitive TIRFM with $100 \times / 1.45$ NA Plan Apochromat objective (Olympus, Japan) and a 14-bit back-illuminated electron-multiplying charge-coupled device camera (EMCCD; Andor iXon DU-897 BV). EGFP was excited at $488 \mathrm{~nm}$ by an argon laser (Melles Griot, Carlsbad, CA, USA). DsRed and Alexa Fluor 555 were exited with the $561 \mathrm{~nm}$ line of laser (Melles Griot). Green and red emissions were collected simultaneously using a dual-view splitter (Optical Insights, Santa Fe, NM, USA) equipped with a 515/30 nm bandpass filter to collect green emission, a $550 \mathrm{~nm}$ dichroic mirror to split the emission, and a $580 \mathrm{~nm}$ long-pass filter to collect the red emission. Videos were acquired using MetaMorph software (Molecular Device).

Live-cell dual-color intracellular tracking was performed with an inverted fluorescence microscope (Olympus IX71, Japan) equipped with a CSU10 spinning-disk confocal laser-scanner unit (Yokogawa Electronic, Tokyo, Japan) and a 100×/1.45NA objective (Olympus). The cells were excited with an argon ion laser (Melles Griot). The fluorescence signals were directed to an EMCCD camera (Andor iXon DU-897 BV). Live-cell triple-color intracellular tracking was performed with the same confocal microscope (Olympus FluoView FV1000, Japan) as described for immunofluorescence.

\section{Image analysis}

Single-particle tracking was performed with Image $J$ (http://rsb. info.nih.gov/ij/). Kymographs of moving objects were generated using ImageJ plugin Multiple Kymograph.

Triple colocalization of Myc-T $\beta$ RI, caveolin-1 and EEA1 was determined by using the Blobprob ImageJ plugin as previously reported [58]. Cells with a medium expression and labeling level of Myc-T $\beta$ RI were selected for each experiment groups. The Mander's overlay coefficients were calculated based on the triple colocalization identified (Supplementary information, Figure S4C). The triple colocalization was also determined by visual inspection. Briefly, for evaluating the colocalizaiton of Myc-T $\beta$ RI with caveolin-1 and EEA1, a low threshold in each channel was used to ensure only the clearly observed monodispersed Myc-T $\beta$ RI, caveolin-1 and EEA1 spots were included in the analysis. After merging the Myc-T $\beta R I$ channel with either caveolin-1 or EEA1 channel by Image $\mathrm{J}$, the colocalization of Myc-T $\beta$ RI with caveolin- 1 or EEA1 was manually counted. Then for each Myc-T $\beta$ RI spot, if it was found to colocalize with both caveolin-1 and EEA1 in the merged channels, it was assigned as the triple-positive vesicle (caveolin-1+EEA1). We found that the Mander's coefficients determined 
by Blobprob ImageJ plugin matched well with the colocalization level determined by visual inspection (Supplementary information, Figure S4).

\section{Electron and immunoelectron microscopy}

Electron microscopy of clathrin-coated pits/vesicles and caveolae in HeLa cells was performed as follows: HeLa cells were fixed with $2.5 \%$ glutaraldehyde in PBS for $24 \mathrm{~h}$. The cells were then post-fixed in $1 \%$ osmium tetroxide for $1 \mathrm{~h}$, embedded in Spurr's resin, sectioned, doubly stained with uranyl acetate and lead citrate, and then examined using a Zeiss EM 10 transmission electron microscope.

Double immunogold microscopy was performed as previously described $[59,60]$. HeLa cells were fixed with $4 \%$ formaldehyde in PBS for $24 \mathrm{~h}$. The cells were then post-fixed in 1\% osmium tetroxide for $1 \mathrm{~h}$, embedded in Spurr's resin and sectioned. The sections were blocked by incubating in PBS containing 5\% BSA, $10 \%$ normal goat serum and $0.1 \%$ cold water fish skin gelatin for $1 \mathrm{~h}$. Then the sections were immunostained with rabbit polyclonal anti-caveolin-1 (610059, BD biosciences, 1:100) in the blocking solution at $4{ }^{\circ} \mathrm{C}$ overnight. The sections were then washed several times with PBS and incubated with 10-nm protein A-gold (provided by Dr Posthuma G, University of Utrecht, The Netherlands) at room temperature for $1 \mathrm{~h}$. The sections were then washed several times with PBS and incubated in the blocking solution at room temperature for $3 \mathrm{~h}$. Then the sections were incubated with rabbit polyclonal anti-EEA1 (sc-33585, Santa Cruz, 1:100) followed by the 15-nm protein A-gold (provided by Dr Posthuma G) as described above. In the control sections, low levels of gold nanoparticle-labeling were detected. The sections were then washed several times with PBS and were doubly stained with uranyl acetate and lead citrate, and examined using a Zeiss EM 10 transmission electron microscope.

\section{Immunoisolation of early endosomes}

The EEA1-positive early endosomes were isolated as previously described $[28,29]$. In brief, the cells were washed with icecold PBS twice, rinsed with osmotic buffer $(10 \mathrm{mM}$ Tris/ $\mathrm{HCl}, \mathrm{pH}$ 7.4) for $1 \mathrm{~min}$, and treated with homogenization buffer $(10 \mathrm{mM}$ Tris/HCl, $1 \mathrm{mM}$ EGTA, $0.5 \mathrm{mM}$ EDTA, $3 \mathrm{mM}$ Imidazole, 250 $\mathrm{mM}$ sucrose, Complete protease inhibitors, $\mathrm{pH}$ 7.4). Then the cells were scraped and collected. The cells were homogenized by 10 passes through a needle. The cell homogenates were centrifuged at $1000 \times g$ at $4{ }^{\circ} \mathrm{C}$ for $10 \mathrm{~min}$ and the postnuclear supernatant was collected. The early endosomes were immunoprecipitated by anti-EEA1 antibodies and protein-A beads or magnetic beads. After the beads were washed with $0.1 \%$ BSA-containing PBS, they were loaded with the loading buffer and boiled. The immunoprecipitated early endosomes were then analyzed by western blotting using specific antibodies.

\section{Statistical analysis}

The Student's two-tailed $t$-test or One-way ANOVA test used in statistical analysis was performed with GraphPad Prism (GraphPad Software). $P<0.05$ was regarded as statistically significant.

\section{Acknowledgments}

We thank Drs Sanford M Simon, Richard D Minshall, Lois E
Greene, Yan Chen, Marino Zerial and Harald Stenmark for their generous gifts of the clathrin-DsRed, caveolin-1-EGFP, EGFP-clathrin, caveolin-ECFP, EGFP-Rab5, ECFP-Rab5, ECFPRab5S34N, EGFP-Rab11, ECFP-Rab11 and Rab5Q79L plasmids. We thank Ying Li (Center of Biomedical Analysis, Tsinghua University) for technical assistance in transmission electron microscopy. This work was supported by the National Basic Research Program of China (2013CB933701), the National Natural Science Foundation of China (91413119, 91213305, 81270159 and 31221064) and the Chinese Academy of Sciences.

\section{References}

1 Mayor S, Pagano RE. Pathways of clathrin-independent endocytosis. Nat Rev Mol Cell Biol 2007; 8:603-612.

2 Aguilar RC, Wendland B. Endocytosis of membrane receptors: two pathways are better than one. Proc Natl Acad Sci USA 2005; 102:2679-2680.

3 Conner SD, Schmid SL. Regulated portals of entry into the cell. Nature 2003; 422:37-44.

4 Pelkmans L, Helenius A. Endocytosis via caveolae. Traffic 2002; 3:311-320.

5 Lu Z, Murray JT, Luo W, et al. Transforming growth factor beta activates Smad2 in the absence of receptor endocytosis. $J$ Biol Chem 2002; 277:29363-29368.

6 Hayes S, Chawla A, Corvera S. TGF beta receptor internalization into EEA1-enriched early endosomes: role in signaling to Smad2. J Cell Biol 2002; 158:1239-1249.

7 Mitchell H, Choudhury A, Pagano RE, Leof EB. Ligand-dependent and -independent transforming growth factor-beta receptor recycling regulated by clathrin-mediated endocytosis and Rab11. Mol Biol Cell 2004; 15:4166-4178.

8 Di Guglielmo GM, Le Roy C, Goodfellow AF, Wrana JL. Distinct endocytic pathways regulate TGF-beta receptor signalling and turnover. Nat Cell Biol 2003; 5:410-421.

9 Feng XH, Derynck R. Specificity and versatility in tgf-beta signaling through Smads. Annu Rev Cell Dev Biol 2005; 21:659-693.

10 Kang JS, Liu C, Derynck R. New regulatory mechanisms of TGF-beta receptor function. Trends Cell Biol 2009; 19:385394.

11 Ikushima H, Miyazono K. TGFbeta signalling: a complex web in cancer progression. Nat Rev Cancer 2010; 10:415424.

12 Massague J. TGFbeta in Cancer. Cell 2008; 134:215-230.

13 Moustakas A, Heldin CH. The regulation of TGFbeta signal transduction. Development 2009; 136:3699-3714.

14 Shi Y, Massague J. Mechanisms of TGF-beta signaling from cell membrane to the nucleus. Cell 2003; 113:685-700.

15 Anders RA, Arline SL, Doré JJ, Leof EB. Distinct endocytic responses of heteromeric and homomeric transforming growth factor beta receptors. Mol Biol Cell 1997; 8:2133-2143.

16 Penheiter SG, Mitchell H, Garamszegi N, Edens M, Doré JJ, Leof EB. Internalization-dependent and -independent requirements for transforming growth factor beta receptor signaling via the Smad pathway. Mol Cell Biol 2002; 22:4750-4759.

17 Runyan CE, Schnaper HW, Poncelet AC. The role of internalization in transforming growth factor beta1-induced Smad2 association with Smad anchor for receptor activation (SARA) 
and Smad2-dependent signaling in human mesangial cells. $J$ Biol Chem 2005; 280:8300-8308.

18 Yao D, Ehrlich M, Henis YI, Leof EB. Transforming growth factor-beta receptors interact with AP2 by direct binding to beta2 subunit. Mol Biol Cell 2002; 13:4001-4012.

19 Razani B, Zhang XL, Bitzer M, von Gersdorff G, Bottinger EP, Lisanti MP. Caveolin-1 regulates transforming growth factor (TGF)-beta/SMAD signaling through an interaction with the TGF-beta type I receptor. J Biol Chem 2001; 276:67276738.

20 Zuo W, Chen YG. Specific activation of mitogen-activated protein kinase by transforming growth factor-beta receptors in lipid rafts is required for epithelial cell plasticity. Mol Biol Cell 2009; 20:1020-1029.

21 Chen YG. Endocytic regulation of TGF-beta signaling. Cell Res 2009; 19:58-70.

22 Nichols B. Caveosomes and endocytosis of lipid rafts. J Cell Sci 2003; 116:4707-4714.

23 Pelkmans L, Burli T, Zerial M, Helenius A. Caveolin-stabilized membrane domains as multifunctional transport and sorting devices in endocytic membrane traffic. Cell 2004; 118:767-780.

24 Querbes W, O'Hara BA, Williams G, Atwood WJ. Invasion of host cells by JC virus identifies a novel role for caveolae in endosomal sorting of noncaveolar ligands. J Virol 2006; 80:9402-9413.

25 Laniosz V, Holthusen KA, Meneses PI. Bovine papillomavirus type 1: from clathrin to caveolin. $J$ Virol 2008; 82:62886298.

26 Sverdlov M, Shinin V, Place AT, Castellon M, Minshall RD. Filamin A regulates caveolae internalization and trafficking in endothelial cells. Mol Biol Cell 2009; 20:4531-4540.

27 Pelkmans L, Zerial M. Kinase-regulated quantal assemblies and kiss-and-run recycling of caveolae. Nature 2005; 436:128-133.

28 Stasyk T, Schiefermeier N, Skvortsov S, et al. Identification of endosomal epidermal growth factor receptor signaling targets by functional organelle proteomics. Mol Cell Proteomics 2007; 6:908-922.

29 Provoda CJ, Waring MT, Buckley KM. Evidence for a primary endocytic vesicle involved in synaptic vesicle biogenesis. $J$ Biol Chem 2000; 275:7004-7012.

30 Sandvig K, Torgersen ML, Raa HA, van Deurs B. Clathrin-independent endocytosis: from nonexisting to an extreme degree of complexity. Histochem Cell Biol 2008; 129:267-276.

31 Hansen CG, Nichols BJ. Molecular mechanisms of clathrin-independent endocytosis. J Cell Sci 2009; 122:1713-1721.

32 Rappoport JZ, Simon SM. Endocytic trafficking of activated EGFR is AP-2 dependent and occurs through preformed clathrin spots. J Cell Sci 2009; 122:1301-1305.

33 McLauchlan H, Newell J, Morrice N, Osborne A, West M, Smythe E. A novel role for Rab5-GDI in ligand sequestration into clathrin-coated pits. Curr Biol 1998; 8:34-45.

34 Zerial M, McBride H. Rab proteins as membrane organizers. Nature Rev Mol Cell Biol 2001; 2:107-117

35 Hagiwara M, Shirai Y, Nomura R, et al. Caveolin-1 activates Rab5 and enhances endocytosis through direct interaction. Biochem Biophys Res Commun 2009; 378:73-78.

36 Panopoulou E, Gillooly DJ, Wrana JL, et al. Early endosomal regulation of Smad-dependent signaling in endothelial cells. $J$ Biol Chem 2002; 277:18046-18052.

37 Tsukazaki T, Chiang TA, Davison AF, Attisano L, Wrana JL. SARA, a FYVE domain protein that recruits Smad2 to the TGFbeta receptor. Cell 1998; 95:779-791.

38 Gagescu R, Demaurex N, Parton RG, Hunziker W, Huber LA, Gruenberg J. The recycling endosome of Madin-Darby canine kidney cells is a mildly acidic compartment rich in raft components. Mol Biol Cell 2000; 11:2775-2791.

39 Zhao B, Wang Q, Du J, Luo S, Xia J, Chen YG. PICK1 promotes caveolin-dependent degradation of TGF-beta type I receptor. Cell Res 2012; 22:1467-1478.

40 Sharma DK, Choudhury A, Singh RD, Wheatley CL, Marks DL, Pagano RE. Glycosphingolipids internalized via caveolar-related endocytosis rapidly merge with the clathrin pathway in early endosomes and form microdomains for recycling. J Biol Chem 2003; 278:7564-7572.

41 Rappoport JZ, Simon SM, Benmerah A. Understanding living clathrin-coated pits. Traffic 2004; 5:327-337.

42 Massol RH, Boll W, Griffin AM, Kirchhausen T. A burst of auxilin recruitment determines the onset of clathrin-coated vesicle uncoating. Proc Natl Acad Sci USA 2006; 103:1026510270 .

43 Abrami L, Liu S, Cosson P, Leppla SH, van der Goot FG. Anthrax toxin triggers endocytosis of its receptor via a lipid raft-mediated clathrin-dependent process. J Cell Biol 2003; 160:321-328.

44 Parton RG, Hanzal-Bayer M, Hancock JF. Biogenesis of caveolae: a structural model for caveolin-induced domain formation. J Cell Sci 2006; 119:787-796.

45 Bates M, Huang B, Dempsey GT, Zhuang X. Multicolor super-resolution imaging with photo-switchable fluorescent probes. Science 2007; 317:1749-1753.

46 Saffarian S, Cocucci E, Kirchhausen T. Distinct dynamics of endocytic clathrin-coated pits and coated plaques. PLoS Biol 2009; 7:e1000191.

47 Parton RG, Simons K. The multiple faces of caveolae. Nat Rev Mol Cell Biol 2007; 8:185-194.

48 Vonderheit A, Helenius A. Rab7 associates with early endosomes to mediate sorting and transport of Semliki forest virus to late endosomes. PLoS Biol 2005; 3:e233.

49 Boyd FT, Massague J. Transforming growth factor-beta inhibition of epithelial cell proliferation linked to the expression of a 53-kDa membrane receptor. J Biol Chem 1989; 264:22722278.

50 Zhang S, Fei T, Zhang L, et al. Smad7 antagonizes transforming growth factor beta signaling in the nucleus by interfering with functional Smad-DNA complex formation. Mol Cell Biol 2007; 27:4488-4499.

$51 \mathrm{Xu} \mathrm{L}$, Chen YG, Massague J. The nuclear import function of Smad2 is masked by SARA and unmasked by TGFbeta-dependent phosphorylation. Nat Cell Biol 2000; 2:559-562.

52 Rappoport JZ, Kemal S, Benmerah A, Simon SM. Dynamics of clathrin and adaptor proteins during endocytosis. $\mathrm{Am} \mathrm{J}$ Physiol Cell Physiol 2006; 291:C1072-C1081.

$53 \mathrm{Wu}$ X, Zhao X, Baylor L, Kaushal S, Eisenberg E, Greene LE. Clathrin exchange during clathrin-mediated endocytosis. J Cell Biol 2001; 155:291-300.

54 Ding Q, Wang Z, Chen Y. Endocytosis of adiponectin receptor 
1 through a clathrin- and Rab5-dependent pathway. Cell Res 2009; 19:317-327.

55 Raiborg C, Wesche J, Malerod L, Stenmark H. Flat clathrin coats on endosomes mediate degradative protein sorting by scaffolding Hrs in dynamic microdomains. J Cell Sci 2006; 119:2414-2424.

56 Hoepfner S, Severin F, Cabezas A, et al. Modulation of receptor recycling and degradation by the endosomal kinesin KIF16B. Cell 2005; 121:437-450.

57 Tagawa A, Mezzacasa A, Hayer A, Longatti A, Pelkmans L, Helenius A. Assembly and trafficking of caveolar domains in the cell: caveolae as stable, cargo-triggered, vesicular trans- porters. J Cell Biol 2005; 170:769-779.

58 Klein ME, Younts TJ, Castillo PE, Jordan BA. RNA-binding protein Sam68 controls synapse number and local beta-actin mRNA metabolism in dendrites. Proc Natl Acad Sci USA 2013; 110:3125-3130.

59 Confalonieri S, Salcini AE, Puri C, Tacchetti C, Di Fiore PP. Tyrosine phosphorylation of Eps 15 is required for ligand-regulated, but not constitutive, endocytosis. J Cell Biol 2000; 150:905-912.

60 Kyei GB, Dinkins C, Davis AS, et al. Autophagy pathway intersects with HIV-1 biosynthesis and regulates viral yields in macrophages. J Cell Biol 2009; 186:255-268.

(Supplementary information is linked to the online version of the paper on the Cell Research website.) 